Pacific Journal of Mathematics

MULTIPLIERS FOR $|C, 1|$ SUMMABILITY OF FOURIER 


\section{MULTIPLIERS FOR $|C, 1|$ SUMMABILITY OF FOURIER SERIES}

\section{G. S. PANDEY}

In the present paper we improve the conditions of all previously known theorems on the absolute $(C, 1)$ summability factors of Fourier series.

1. Let the formal expansion of a function $f(x)$, periodic with period $2 \pi$ and integrable in the sense of Lebesgue over $[-\pi, \pi]$, in a Fourier-trigonometric series be given by

$$
f(x) \sim \frac{1}{2} a_{0}+\sum_{n=1}^{\infty}\left(a_{n} \cos n x+b_{n} \sin n x\right) \equiv \sum_{n=0}^{\infty} A_{n}(x) .
$$

We write

$$
\phi(u)=f(x+u)+f(x-u)-2 f(x)
$$

and throughout this paper $A$ will denote a positive constant, not necessarily the same at each occurrence.

Whittaker [5], in 1930, proved that the series

$$
\sum_{n=1}^{\infty} A_{n}(x) / n^{\alpha}, \quad \alpha>0,
$$

is summable $|A|$ almost everywhere.

Later, Prasad [4] demonstrated that the series

$$
\sum_{n=n_{0}}^{\infty} A_{n}(x) / \mu_{n},
$$

where

$$
\mu_{n}=\left(\prod_{\nu=1}^{k-1} \log ^{\nu} n\right)\left(\log ^{k} n\right)^{1+\varepsilon}, \quad \log ^{k} n_{0}>0, \quad \varepsilon>0,
$$

and

$$
\log ^{k} n=\log \left(\log ^{k-1} n\right), \cdots, \log ^{2} n=\log \log n ;
$$

is summable $|A|$ almost everywhere.

Chow [2], on the other hand, has shown that the series $\sum \lambda_{n} A_{n}(x)$ is summable $|C, 1|$ almost everywhere, provided $\left\{\lambda_{n}\right\}$ is a convex sequence satisfying the condition $\sum n^{-1} \cdot \lambda_{n}<\infty$.

Cheng [1], in 1948, established the following: 


$$
\Phi(t) \equiv \int_{0}^{t}|\phi(u)| d u=O(t)
$$

as $t \rightarrow 0$, then the series

$$
\sum_{n=2}^{\infty} A_{n}(x) /(\log n)^{1+\delta}, \quad \delta>0
$$

is summable $|C, \alpha|, \alpha>1$.

In a recent paper, Hsiang [3] has proved the following theorems:

THEOREM B. If

$$
\Phi(t)=O(t) \quad(t \longrightarrow+0),
$$

then the series $\sum_{n=1}^{\infty} A_{n}(x) / n^{\alpha}$ is summable $|C, 1|$ for every $\alpha>0$.

Theorem C. If

$$
\Phi(t)=O\left\{t / \prod_{\nu=1}^{k} \log ^{\nu}(1 / t)\right\}
$$

as $t \rightarrow+0$, then the series

$$
\sum_{n=0}^{\infty} A_{n}(x) /\left(\prod_{\nu=1}^{k-1} \log ^{\nu} n\right)\left(\log ^{k} n\right)^{1+\varepsilon}
$$

is summable $|C, 1|$ for every $\varepsilon>0$.

In the present paper we prove the following theorem, which includes the theorem of Cheng and both the theorems of Hsiang:

THEOREM. If

$$
\varphi(t) \equiv \int_{t}^{o} \frac{|\phi(u)|}{u} d u=O\left\{\left(\log ^{k}(1 / t)\right)^{\eta}\right\} \quad \text { as } \quad t \longrightarrow+0,
$$

$0<\delta \leqq \pi$, then the series (1.4) is summable $|C, 1|$ for $0<\eta<\varepsilon$.

The conditions of our theorem are less stringent than those of Cheng and Hsiang.

2. The proof of the theorem is based on the following lemmas:

Lemma 1. Let $S_{n}(x)$ be the $n$th partial sum of the series (1.1), then under the condition (1.5), we have

$$
\sum_{\nu=0}^{n}\left|S_{\nu}(x)-f(x)\right|=O\left\{n\left(\log ^{1 /} n\right)^{\eta}\right\} .
$$


Proof. Let $\varepsilon_{\nu}=\operatorname{sign}\left[S_{\nu}(x)-f(x)\right]$, so that $\varepsilon_{\nu}= \pm 1$ and it depends only upon $x$ and $\nu$, and is independent of $t$. Also, we write

$$
K_{n}(t)=\sum_{\nu=0}^{n} \varepsilon_{\nu} \sin \nu t
$$

Thus, we have

$$
\begin{aligned}
\sum_{j=0}^{n}\left|S_{v}(x)-f(x)\right| & =\frac{2}{\pi} \int_{0}^{\pi} \frac{\phi(t)}{t} K_{n}(t) d t+o(n) \\
& =\frac{2}{\pi}\left[\int_{0}^{1 / n}+\int_{1 / n}^{\delta}+\int_{\delta}^{\pi}\right]+o(n) \\
& =I_{1}+I_{2}+I_{3}+o(n),
\end{aligned}
$$

say. Now,

$$
\begin{aligned}
I_{1} & \leqq \int_{0}^{1 / n}|\phi(t)| \cdot O\left(n^{2}\right) d t \\
& =O\left(n^{2}\right) \int_{0}^{1 / n}-t \phi^{\prime}(t) d t, \quad \varphi^{\prime}(t)=\frac{d}{d t} \varphi(t) . \\
& =O\left(n^{2}\right)[-t \varphi(t)]_{0}^{1 / n}+O\left(n^{2}\right) \int_{0}^{1 / n} \varphi(t) d t \\
& =O\left\{n\left(\log ^{k} n\right)^{\eta}\right\} .
\end{aligned}
$$

Also, for $n t \geqq 1$, we have

$$
\begin{aligned}
I_{2} & \leqq \int_{1 / n}^{\delta} \frac{|\phi(t)|}{t} \cdot n d t \\
& =O\left\{n\left(\log ^{k} n\right)^{\eta}\right\} .
\end{aligned}
$$

Since, by Riemann-Lebesgue theorem,

$$
\int_{\delta}^{\pi} \frac{\phi(t)}{t} \sin n t d t=o(1)
$$

we have

$$
I_{3}=O(n) \text {. }
$$

Combining (2.1), (2.2), $\cdots,(2.5)$, the lemma follows.

LEMMA 2. Let

$$
t_{n}(x)=\frac{1}{(n+1)} \sum_{\nu=1}^{n} \nu A_{\nu}(x)
$$

Then

$$
T_{n}(x) \equiv \sum_{\nu=1}^{n}\left|t_{\nu}(x)\right|=O\left\{n\left(\log ^{k} n\right)^{\eta}\right\}
$$


and

$$
\sum_{n=n_{0}}^{\infty}\left(\mu_{n}\right)^{-1} \cdot n^{-1}\left|t_{\nu}(x)\right|<\infty
$$

Proof. Let

$$
\sigma_{n}(x)=\frac{1}{(n+1)} \sum_{\nu=0}^{n} S_{\nu}(x)
$$

Thus, we have

$$
\begin{aligned}
\sigma_{n}(x)-f(x)= & \frac{1}{(n+1)} \sum_{\nu=0}^{n}\left\{S_{\nu}(x)-f(x)\right\} \\
\Longrightarrow\left|\sigma_{n}(x)-f(x)\right| & \leqq \frac{1}{(n+1)} \sum_{\nu=0}^{n}\left|S_{\nu}(x)-f(x)\right| \\
& =O\left\{\left(\log ^{k} n\right)^{\eta}\right\}
\end{aligned}
$$

by Lemma 1.

Therefore, we find that

$$
\begin{aligned}
T_{n}(x) & =\sum_{\nu=1}^{n}\left|t_{\nu}(x)\right| \\
& =\sum_{\nu=1}^{n}\left|S_{\nu}(x)-\sigma_{\nu}(x)\right| \\
& \leqq \sum_{\nu=1}^{n}\left|S_{\nu}(x)-f(x)\right|+\sum_{\nu=1}^{n}\left|\sigma_{\nu}(x)-f(x)\right| \\
& =O\left[n\left(\log ^{k} n\right)^{\eta}\right]
\end{aligned}
$$

by (2.6) and Lemma 1.

Finally, by Abel's transformation, we have

$$
\begin{aligned}
\sum_{n=m}^{M}\left(\mu_{n}\right)^{-1} \cdot n^{-1}\left|t_{n}(x)\right|= & \sum_{n=m}^{M-1} T_{n}(x) \Delta\left\{\left(\mu_{n}\right)^{-1} \cdot n^{-1}\right\} \\
& -\left(\mu_{m-1}\right)^{-1}(m-1)^{-1} T_{m-1}(n)+\mu_{M}^{-1} \cdot M^{-1} T_{M}(x) \\
= & \sum_{n=m}^{M-1} \Delta\left\{\left(\mu_{n}\right)^{-1}\right\} \cdot n^{-1} T_{n}(x) \\
& +\sum_{m=m}^{M-1}\left(\mu_{n+1}\right)^{-1} \cdot n^{-1}(n+1)^{-1} T_{n}(x)+O(1) \\
= & \sum_{n=m}^{M-1} \Delta\left\{\left(\mu_{n}\right)^{-1}\right\} \cdot\left(\log ^{k} n\right)^{\eta} \\
& +\sum_{n=m}^{M-1}\left(\mu_{n+1}\right)^{-1}(n+1)^{-1}\left(\log ^{k} n\right)^{\eta}+O(1) \\
\leqq & \sum_{n=m}^{M-1} \frac{A \cdot\left(\log ^{k} n\right)^{\eta}}{n\left(\prod_{\mu=1}^{k-1} \log ^{\mu} n\right)\left(\log ^{k} n\right)^{1+\varepsilon}}+O(1) \\
= & O(1),
\end{aligned}
$$


for $m \rightarrow \infty$ and $M \rightarrow \infty$.

In view of (2.7) and (2.8) the lemma is proved.

3. Proof of the theorem. Let $\tau_{n}(x)$ denotes the $n$th Cesàro mean of the sequence $\left\{n\left(\mu_{n}^{-1}\right) \cdot A_{n}(x)\right\}$.

By Abel's transformation, we have

$$
\begin{aligned}
\tau_{n}(x) & =\frac{1}{(n+1)} \sum_{\nu=n_{0}}^{n} \nu\left(\mu_{\nu}\right)^{-1} \cdot A_{\nu}(x) \\
& =\frac{1}{(n+1)} \sum_{\nu=n_{0}}^{n-1} \Delta\left(\mu_{\nu}\right)^{-1} \cdot(\nu+1) t_{\nu}(x)+\left(\mu_{n}\right)^{-1} t_{n}(x) \\
& =J_{1}^{(n)}(x)+J_{2}^{(n)}(x),
\end{aligned}
$$

say. Now, by Lemma 2, we find that

$$
\begin{aligned}
\sum_{n=m_{0}}^{m} J_{1}^{(n)}(x) / n & \leqq \sum_{n=m_{0}}^{m} n^{-1}(n+1)^{-1} \sum_{\nu=n_{0}}^{n-1} \Delta\left(\mu_{\nu}\right)^{-1}(\nu+1)\left|t_{\nu}(x)\right|, \quad \log ^{k} m_{0}>0 \\
& \leqq A \sum_{\nu=m_{0}}^{m} \Delta\left(\mu_{\nu}\right)^{-1}(\nu+1)\left|t_{\nu}(x)\right| \sum_{n=\nu+1}^{m} n^{-1}(n+1)^{-1} \\
& \leqq A \sum_{\nu=m_{0}}^{m} \Delta\left(\mu_{\nu}\right)^{-1}\left|t_{\nu}(x)\right| \\
& =A \sum_{\nu=m_{0}}^{m-1} \Delta^{2}\left[\left(\mu_{\nu}\right)^{-1}\right] \cdot T_{\nu}(x)+\Delta\left(\mu_{m}^{-1}\right) T_{m}(x)+O(1) \\
& =O(1) .
\end{aligned}
$$

Also, we have

$$
\begin{aligned}
\sum_{n=m_{0}}^{m} J_{2}^{(n)}(x) / n & \leqq \sum_{n=m_{0}}^{m}\left(\mu_{n}\right)^{-1} \cdot n^{-1} t_{n}(x) \mid \\
& =O(1) .
\end{aligned}
$$

From (3.1), (3.2), and (3.3), we have

$$
\sum_{n=m_{0}}^{m} \frac{\left|\tau_{n}(x)\right|}{n}=O(1) \text {. }
$$

This completes the proof of the theorem.

\section{REFERENCES}

1. M. T. Cheng, Summability factors of Fourier series, Duke Math. J., 15 (1948), $17-27$.

2. H. C. Chow, On the summability factors of Fourier series, J. London Math. Soc., 16 (1941), 215-220.

3. F. C. Hsiang, On C, I summability factors of Fourier series at a given point, Pacific J. Math., 33 (1970), 139-147.

4. B. N. Prasad, On the summability of Fourier series and the bounded variation of power series, Proc. London Math. Soc., (2), 35 (1933), 407-424. 
5. J. M. Whittaker, The absolute summability of Fourier series, Proc. Edinburgh Math. Soc., (2), 2 (1930), 1-5.

Received January 16, 1975 and in revised form April 3, 1978.

MOSUL UNIVERSITY

MosUl, IRAQ 


\section{PACIFIC JOURNAL OF MATHEMATICS}

EDITORS

RICHARD ARENS (Managing Editor)

University of California

Los Angeles, California 90024

C. W. Curtis

University of Oregon

Eugene, OR 97403

C. C. MOORE

University of California

Berkeley, CA 94720
J. DUGUNDJI

Department of Mathematics University of Southern Californı Los Angeles, California 90007

R. Finn and J. Milgram Stanford University Stanford, California 94305

\section{ASSOCIATE EDITORS}

E. F. BECKENBACH

B. H. NEUMANN

F. WOLF

K. YoSHIDA

\section{SUPPORTING INSTITUTIONS}

UNIVERSITY OF BRITISH COLUMBIA
CALIFORNIA INSTITUTE OF TECHNOLOGY
UNIVERSITY OF CALIFORNIA
MONTANA STATE UNIVERSITY
UNIVERSITY OF NEVADA, RENO
NEW MEXICO STATE UNIVERSITY
OREGON STATE UNIVERSITY
UNIVERSITY OF OREGON

UNIVERSITY OF SOUTHERNEALIFORNIA STANFORD UNIVERSITY UNIVERSITY OF HAWAII UNIVERSITY OF TOKYO UNIVERSITY OF UTAH WASHINGTON STATE UNIVERSITY UNIVERSITY OF WASHINGTON 


\section{Pacific Journal of Mathematics \\ Vol. 79 , No. 1 \\ May, 1978}

Teófilo Abuabara, A remark on infinitely nuclearly differentiable

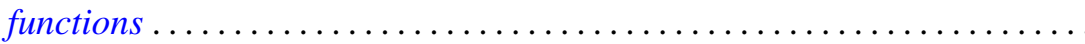

David Fenimore Anderson, Projective modules over subrings of $k[X, Y]$

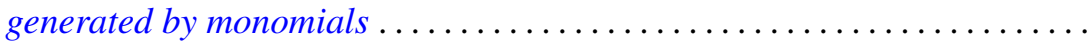

Joseph Barback and Thomas Graham McLaughlin, On the intersection of

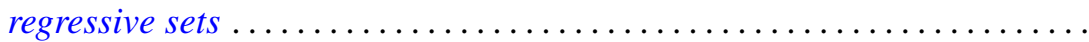

Murray Bell, John Norman Ginsburg and R. Grant Woods, Cardinal inequalities for topological spaces involving the weak Lindelof

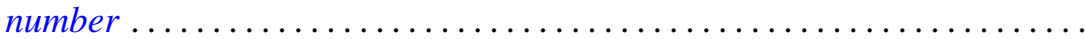

Laurence Richard Boxer, The space of ANRs of a closed surface ............

Zvonko Cerin, Homotopy properties of locally compact spaces at

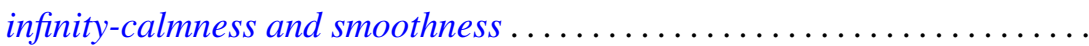

Isidor Fleischer and Ivo G. Rosenberg, The Galois connection between partial functions and relations..................................

John R. Giles, David Allan Gregory and Brailey Sims, Geometrical implications of upper semi-continuity of the duality mapping on a Banach

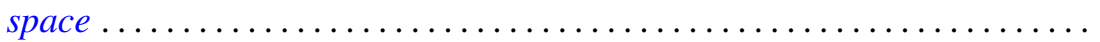

Troy Lee Hicks, Fixed-point theorems in locally convex spaces ............ Hugo Junghenn, Almost periodic functions on semidirect products of transformation semigroups ........................

Victor Kaftal, On the theory of compact operators in von Neumann algebras. II . . . .

Haynes Miller, A spectral sequence for the homology of an infinite

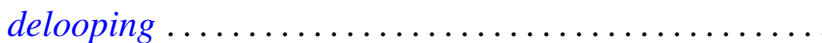

Sanford S. Miller, Petru T. Mocanu and Maxwell O. Reade, Starlike integral operators...

Stanley Stephen Page, Regular FPF rings ...............

Ghan Shyam Pandey, Multipliers for C, 1 summability of Fourier series ...

Shigeo Segawa, Bounded analytic functions on unbounded covering surfaces...

Steven Eugene Shreve, Probability measures and the C-sets of

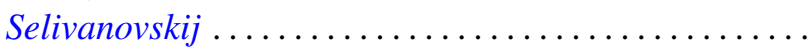

Tor Skjelbred, Combinatorial geometry and actions of compact Lie groups....

Alan Sloan, A note on exponentials of distributions.

Colin Eric Sutherland, Type analysis of the regular representation of a nonunimodular group.

Mark Phillip Thomas, Algebra homomorphisms and the functional

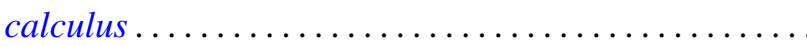

Sergio Eduardo Zarantonello, A representation of $H^{p}$-functions with

$0<p<\infty$. 